\title{
Novo Trabalho: desenvolvendo narrativas críticas sobre o trabalho na pandemia
}

\author{
Novo Trabalho: developing critical narratives about work in the pandemic
}

Antônio Vinícius Dantas Moura de Oliveira, Eriadne Teixeira do Nascimento,

Ewerton Bezerra Siqueira de Miranda, Rafael de Lima Cardozo,

Helena Rugai Bastos, Luiza Falcão Soares da Cunha

dingbat, COVID-19, trabalhadores

O artigo apresenta o projeto da fonte Novo Trabalho, realizado na disciplina Desenho de Tipos, componente curricular do curso Bacharelado em Design da Universidade Federal do Rio Grande do Norte. A proposta foi projetar uma fonte dingbat a partir do tema COVID-19. O projeto buscou explorar o desenvolvimento de um dingbat, que pudesse ser utilizado como ferramenta comunicacional e de crítica. A experiência resultou em uma fonte não-alfabética, que possibilita a construção de narrativas sobre os riscos que caracterizam o cotidiano dos trabalhadores das categorias mais vulneráveis à COVID-19.

dingbat, covid-19, workers

The paper addresses the Novo Trabalho font project, carried out for the Design of types discipline, an optional curricular component of the course Bachelor of Design of the Federal University of Rio Grande do Norte. The proposal was to design a dingbat font inspired by the COVID-19 pandemic. The project sought to explore the design of a dingbat font, which could be used as a communicational and critical instrument. The experience resulted in a non-alphabetical font, in which the relation between the glyphs enables to idealize narratives about the risks that characterize the daily lives of workers in the categories most vulnerable to COVID-19.

\section{Introdução}

Em meio ao atual cenário da pandemia da COVID-19, percebe-se uma mudança na dinâmica de vida da sociedade, tanto no âmbito pessoal quanto profissional. O distanciamento social, principal recomendação para diminuir a proliferação do vírus, não foi acatada de maneira uniforme no Brasil. Essa questão afetou diretamente trabalhadores de serviços essenciais e, também, dos serviços informais.

Entendendo que o design não é apenas uma ferramenta mercadológica, mas que também funciona como instrumento social e político, surgem reflexões sobre como o designer pode atuar nesse contexto. Tendo em mente as inúmeras formas de comunicação possíveis, podemos observar na linguagem não-verbal, o uso de fontes com conjunto de caracteres não-

Anais do $10^{\circ} \mathrm{CIDI}$ e $10^{\circ} \mathrm{CONGIC}$

Kelli C.A.S. Smythe, Rafael de Castro Andrade (orgs.)

Sociedade Brasileira de Design da Informação - SBDI

Curitiba | Brasil | 2021
Proceedings of the $10^{\text {th }} \mathrm{CIDI}$ and $10^{\text {th }} \mathrm{CONGIC}$

Kelli C.A.S. Smythe, Rafael de Castro Andrade (orgs.)

Sociedade Brasileira de Design da Informação - SBDI Curitiba | Brazil | 2021 
alfabéticos, conhecidas como fontes dingbats. Por vezes utilizadas para ornamentação, os dingbats podem, também, funcionar como principal recurso gráfico para a composição de informações.

Esse artigo relata o desenvolvimento de uma fonte dingbat na disciplina Desenho de Tipos do curso Bacharelado em Design da Universidade Federal do Rio Grande do Norte relacionada ao tema COVID-19. Buscou-se representar, por meio de caracteres não-alfabéticos, a rotina dos trabalhadores durante a pandemia, e ressaltar os riscos aos quais estão expostos.

\section{Sobre dingbats}

Monteiro Borges (2011) destaca que tradicionalmente muitas fontes representam caracteres alfabéticos, e uma das funções destas tipografias é possibilitar a leitura do texto escrito. Para além das fontes de texto, Monteiro Borges (2011) apresenta mais dois grupos de tipografias: as decorativas, que conservam relação formal com o desenho de caracteres alfabéticos, e as fontes dingbats, cujos glifos podem ser ornamentos, símbolos, pictogramas sem relação imediata com caracteres alfabéticos.

Considerando o desenvolvimento da tipografia, tais elementos não alfabéticos foram incorporados como recursos gráficos às oficinas tipográficas (Farias, 2001). Farias (2001) destaca que a organização destes elementos em conjuntos de glifos, seguindo o padrão das fontes alfabéticas, foi viabilizado com a tecnologia digital. A partir de então, houve um crescimento expressivo da diversidade de fontes formadas por conjunto de glifos que configuram formas visuais sem relação imediata com caracteres alfabéticos: as fontes dingbats. Apesar da incongruência com os caracteres alfabéticos e da distância formal com o desenho habitual das letras (Cunha, 2019), os projetos de dingbats requerem processo de concepção, de seleção e de edição para estabelecer articulação e coerência formal entre os glifos (Farias, 2001; Cunha, 2019).

\section{Design Crítico e a pandemia da COVID-19}

Segundo DiSalvo (2012, p.3), especialmente a partir do final dos anos 1990, cresceu o interesse em projetos de design vinculados a programas políticos e sociais, ampliando a articulação entre design e inovação social, design e democracia, como formas de "engajamento cívico", o que proporcionou melhoria de processos de governança. Para DiSalvo (2012), essa tendência no design representou postura crítica diante o projeto de artefatos.

Para Eira e Brandalise (2019, p. 29) muitos profissionais e pesquisadores buscam hoje, por meio do design e a partir de esforço coletivo, "pensar o mundo atual" com intuito de viabilizar um futuro para a sociedade. Trata-se de uma postura crítica em relação às oportunidades de projeto, para além de uma orientação mercadológica. É nesse sentido que as abordagens do design crítico se assentam, como a proposta de DiSalvo (2012). O autor acredita na produção 
cultural contemporânea de design como uma construção teórica, para o pensamento e a prática do design.

O termo design crítico é atribuído aos pesquisadores Anthony Dunne e Fiona Raby (DiSalvo, 2012), que nos anos 1990, projetaram artefatos questionando as ordens social, econômica e cultural. Dunne e Raby (2013) explicam que o conceito de design crítico, afirma uma postura crítica, promove a reflexão e o debate que contrapõe a produção de design orientada para o mercado. Considerando, então, uma visão mais crítica do design é possível refletir sobre o atual contexto de pandemia causado pela Covid-19.

Durante a crise da COVID-19, o Brasil vem apresentando políticas dissonantes daquelas recomendadas pela Organização Mundial da Saúde, com destaque para a ausência de ações de isolamento e proteção social. Segundo Rocha et al. (2021), a propagação do vírus está relacionada à vulnerabilidade socioeconômica dos brasileiros. Esta estatística está diretamente ligada às condições socioeconômicas da população brasileira, em especial de trabalhadores, informais ou não, que não puderam aderir ao isolamento social permanecendo em casa.

Em relatório sobre saúde, vulnerabilidade e riscos dos trabalhadores na pandemia causada pela Covid-19, publicado em maio de 2020, a Rede CoVida argumenta que a desregulamentação do trabalho em curso no Brasil nos últimos anos, vem diminuindo os direitos trabalhistas e ampliando a instabilidade dos contratos de trabalho, a subdivisão de categorias e a terceirização das atividades. As recentes reformas trabalhistas, outrossim, sujeitaram os trabalhadores a longas jornadas laborais e os expuseram à falta de assistência à saúde. Esse quadro foi agravado pela pandemia, uma vez que o distanciamento social e a interrupção momentânea de atividades econômicas fizeram parte da estratégia para contenção da COVID-19, mas não foi seguido por diversos trabalhadores formais e informais. De fato, os trabalhadores da saúde sempre estiveram mais expostos aos riscos da COVID-19, entretanto, para este grupo, o diagnóstico periódico e o acesso aos cuidados, EPIs por exemplo, os diferencia de outros trabalhadores, em especial aqueles que se dedicam aos serviços essenciais (Rede CoVida, 2020).

Nesse sentido, o projeto da fonte dingbat Novo Trabalho se apresenta como uma opção de elementos visuais, que podem representar algumas das situações que os trabalhadores mais vulneráveis enfrentam diariamente na pandemia.

\section{A fonte}

O projeto da fonte Novo Trabalho foi desenvolvido no segundo semestre de 2020 , na disciplina (omitido), do curso Bacharelado em Design da Universidade Federal do Rio Grande do Norte e ministrado pelas professoras Luiza Falcão Soares da Cunha e Helena Rugai Bastos de forma remota. A disciplina apresentou como proposta a criação de uma fonte dingbat a partir da metodologia proposta por Cunha (2019) sobre o tema da pandemia da COVID-19. O processo projetual foi dividido em quatro etapas, relatadas a seguir.

Na primeira etapa do projeto, foi elaborada uma pesquisa iconográfica a respeito do tema. Com base na categorização e seleção das referências gráficas, definiu-se como partido 
projetual da fonte a representação dos riscos aos quais os trabalhadores estão sujeitos em meio a pandemia da COVID-19 e a possibilidade de construção de diferentes narrativas a partir dos caracteres desenvolvidos.

Na segunda etapa foram elaborados os esboços a mão livre e, em seguida, definiu-se as relações de proporção vertical, proporção horizontal, espessura das hastes e as regras de funcionamento dos glifos caixa-alta e caixa-baixa. Foram desenhados, ainda, caracteres que serviram de base para a configuração dos demais glifos da fonte.

$\mathrm{Na}$ etapa seguinte, de desenho vetorial, foi construído o grid modular de $1000 \times 1000$ pt, que serviu de base para a construção digital de cada glifo. Por fim, na última etapa, o desenho dos caracteres foi transposto para um software de geração de fontes. A fase incluiu definições de espacejamentos laterais, os ajustes finais nos desenhos dos caracteres, e o fechamento do arquivo da fonte. A fase incluiu definições de espacejamentos laterais, os ajustes finais nos desenhos dos caracteres, e o fechamento do arquivo da fonte.

Figura 1: Grid.

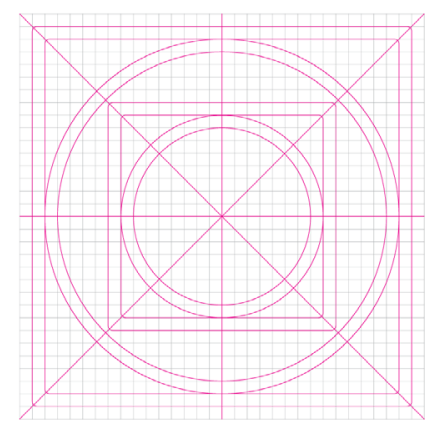

Grid

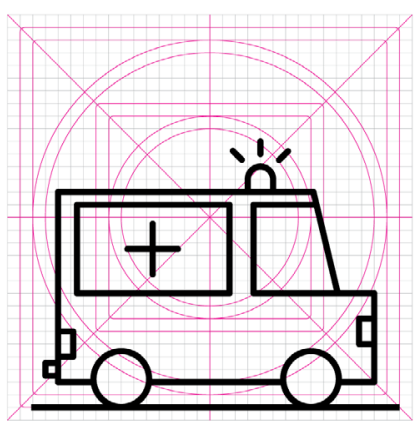

Ícone

\section{Dingbat Novo Trabalho}

Considerando o contexto da pandemia, a importante transformação de hábitos e da rotina da sociedade e na vida dos trabalhadores, a fonte Novo Trabalho foi projetada com o objetivo de representar como a pandemia afeta determinadas parcelas da população em maior proporção. O nome "Novo Trabalho" faz referência direta ao termo "novo normal", comumente usado para passar uma falsa noção da normalidade. Deste modo, a fonte busca trazer uma reflexão sobre esse "normal" em que vivem os trabalhadores informais e de serviços essenciais durante a crise da COVID-19, buscando representar a circulação deste grupo na cidade e algumas situações rotineiras que podem enfrentar.

A fonte consiste em um conjunto de caracteres divididos em seis grupos: Pessoas, Ambientes, Meios de transportes, interações, corona e especiais (Figura 2). Seus caracteres apresentam uma linha de base aparente, que os une, conferindo linearidade à composição gráfica, e permite a criação de diferentes narrativas sequenciais sobre as rotinas de algumas profissões. 
Figura 2: Os seis grupos subdivididos.

Pessoas

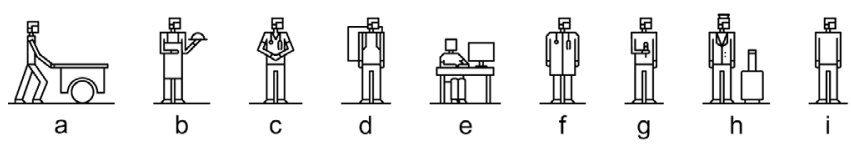

Meios de Transporte

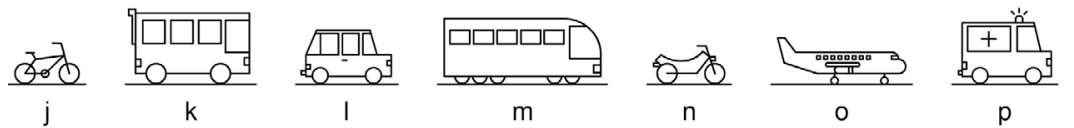

Ambientes

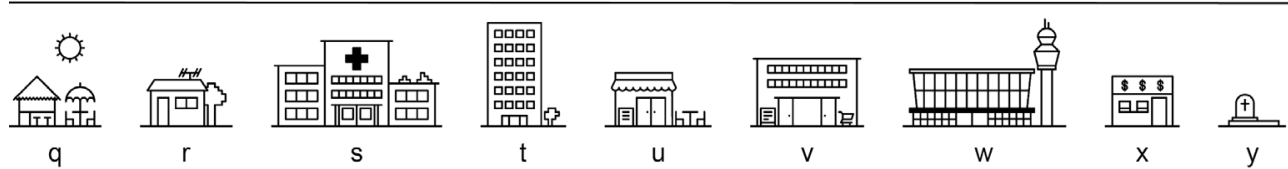

Interações
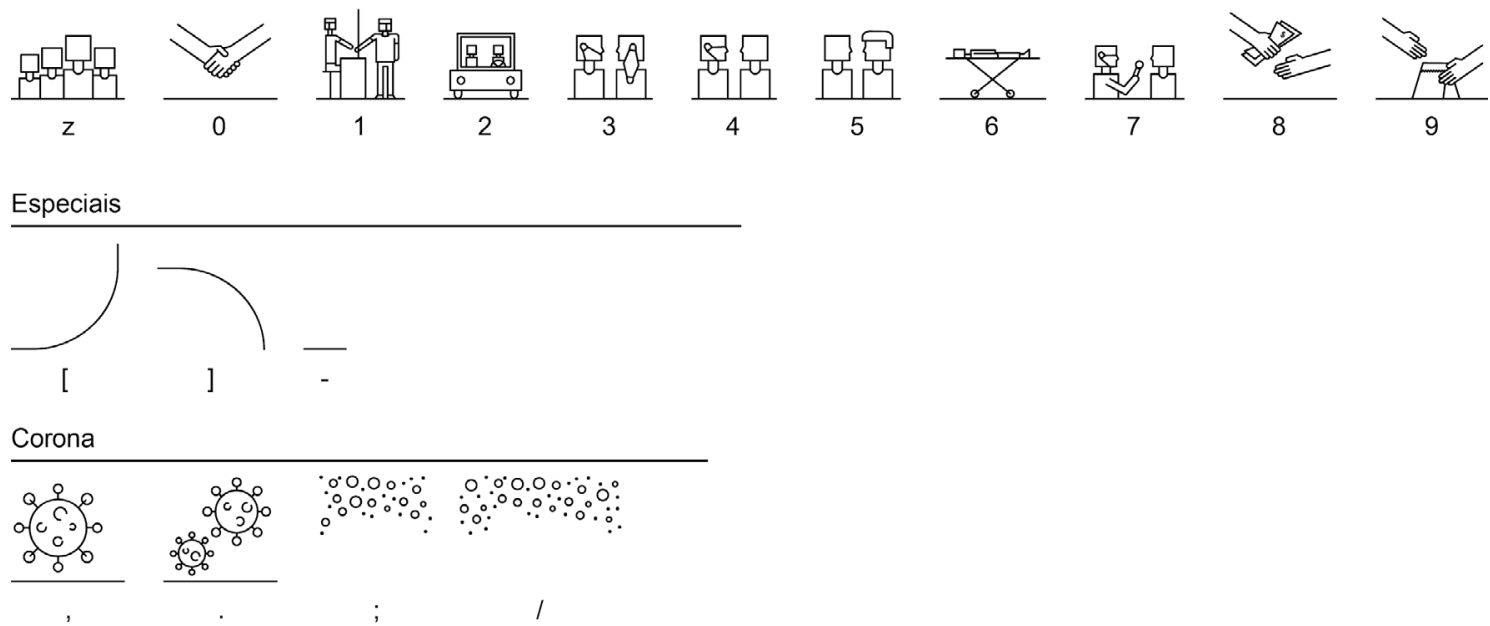

O conjunto da caixa-baixa da fonte (letras e alguns sinais gráficos) possibilita a construção de narrativas da esquerda para a direita, enquanto a caixa-alta corresponde à construção de narrativas da direita para a esquerda (Figura 3). Significa que para inversão da narrativa, basta acionar a tecla Shift ou manter acionada a tecla Caps Lock. 
Figura 3: Relação entre caixa-baixa e caixa-alta.

Caixa-baixa $\longrightarrow$
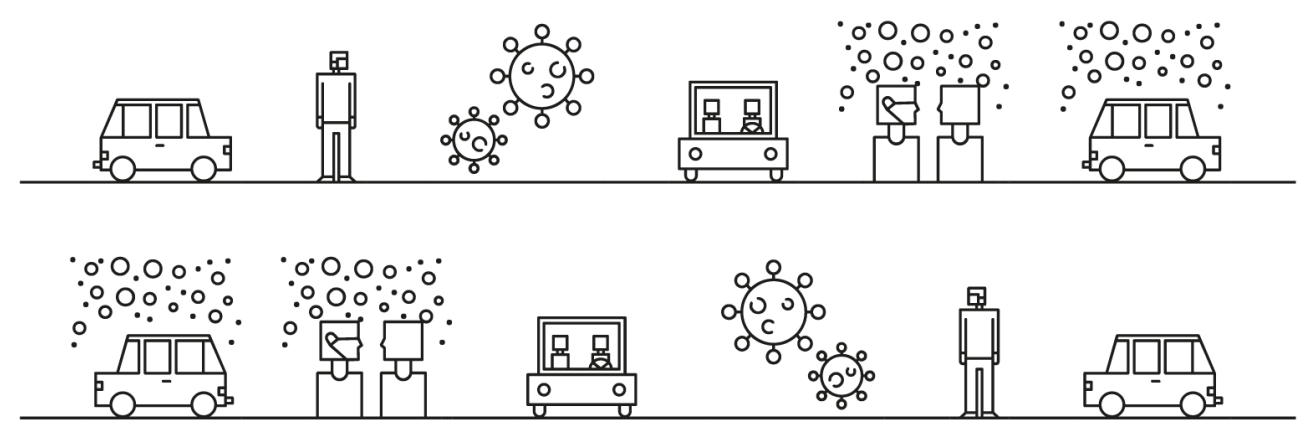

$\longleftarrow$ Caixa-alta

Para auxiliar na composição gráfica de uma narrativa, a fonte oferece no caractere correspondente ao "-" (hífen) uma linha base extra.

Figura 3: Exemplo de utilização do hífen, que representa uma linha de base extra.

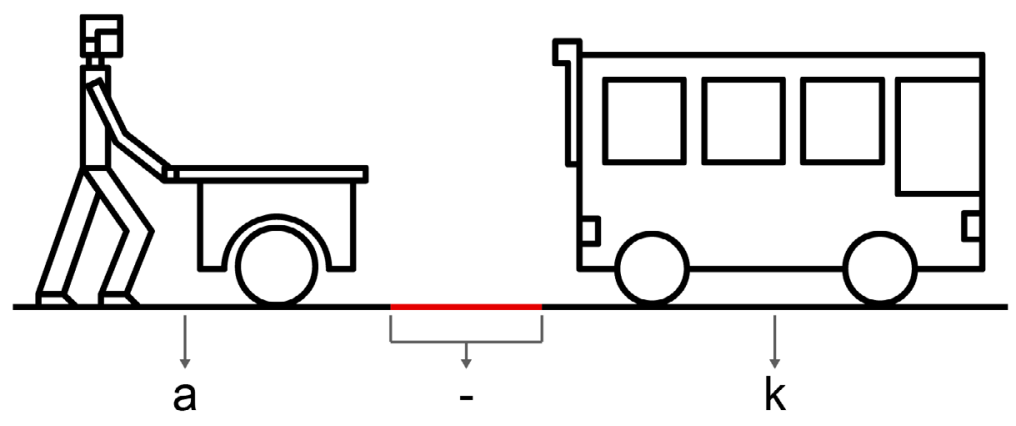

Para possibilitar a construção de narrativas com diferentes orientações, foram projetados desenhos de conexão, os glifos especiais, que correspondem aos sinais "[" (abre colchete) e "]" (fecha colchete) do teclado. Esses desenhos podem ser utilizados para dar sequência à narrativa em diferentes linhas de texto. É importante ressaltar que o uso dos caracteres de conexão deve estar atrelado à mudança de caracteres maiúsculos para minúsculos e viceversa, para que se possa inverter o sentido da narrativa (Figura 5). 
Figura 5: Exemplo de utilização dos sinais "[" (abre colchete) e "]" (fecha colchete).

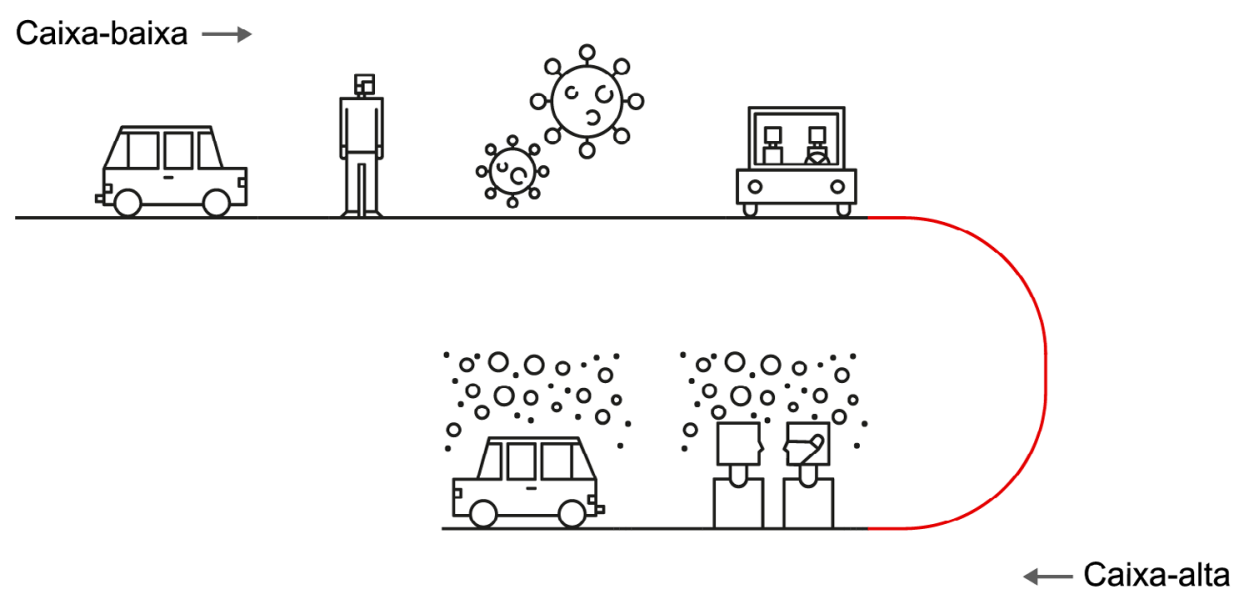

Como mencionado, grande parte dos glifos possui uma linha de base comum, porém dois caracteres da categoria "Corona" não seguem essa regra. Os desenhos representados pelos caracteres ";" (ponto e vírgula) e "." (ponto final), retratam o vírus e a contaminação pelo vírus, e podem ser usados de forma sobreposta. Para tanto, basta digitar qualquer letra ou algarismo seguido do “;”, por exemplo (Figura XX).

Figura 7: Exemplo dos ícones sobrepostos.

$-\mathrm{l-i}-.-2-4$

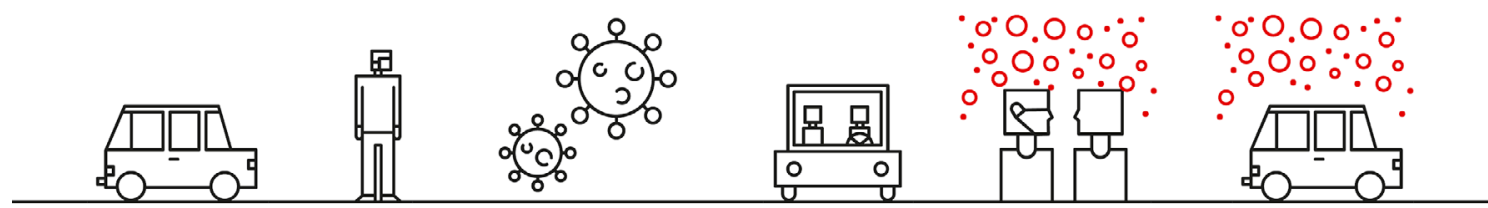

Tendo em vista que nem todos os caracteres possuem proporções horizontais iguais, pode ocorrer falha ao fazer uso dos caracteres de conexão e sobreposição.Para ajuste é possível acrescentar glifos Para ajuste é possível acrescentar glifos "_" (underline) e "-" (hífen) do teclado (Figura 6). É importante destacar que, apesar da fonte ter sido projetada especialmente para a construção de narrativas, seus caracteres podem ser utilizados individualmente. 
Figura 6: Exemplo de utilização “_” (underline) e “-” (hífen) como ajuste.

$-\mid-\mathrm{i}-.-2]$

$-\mathrm{L}$

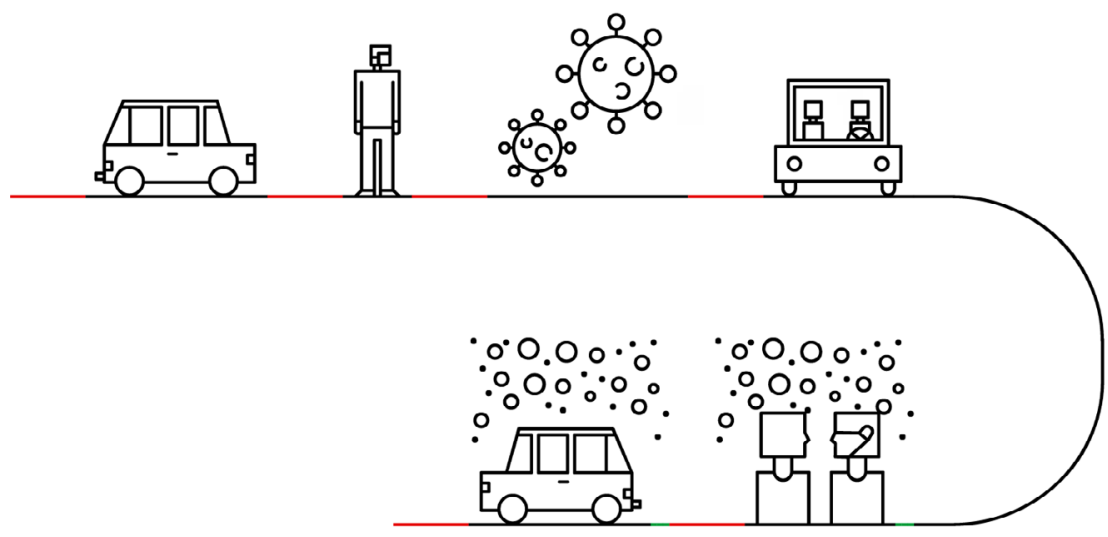

\section{Considerações finais}

O presente trabalho apresentou conceitos acerca das fontes dingbats e sobre o design como ferramenta para reflexão, crítica e debate sobre problemas que enfrentamos diariamente. Neste caminho, o projeto da fonte dingbat Novo Trabalho busca representar algumas situações da realidade dos trabalhadores brasileiros no contexto da pandemia da COVID-19. Pode-se, por meio da fonte, apresentar alguns contextos do cotidiano destes trabalhadores e algumas interações que podem levar à contaminação dessas pessoas. Desta maneira, esta fonte pode ser utilizada como recurso gráfico em peças de comunicação, para enfatizar ou para apoiar a informação em projetos gráficos sobre a pandemia.

Por fim, vale salientar a importância do design em momentos de crise, que pode ir além da função mercadológica e atuar como um agente social poderoso. Neste sentido, a fonte Novo Trabalho pode contribuir para apresentar a realidade que enfrentamos no cenário atual da pandemia pela COVID-19.

\section{Referências}

Cunha, L. F. S. (2019). O desenvolvimento de fontes dingbats como ferramenta para a aprendizagem do processo projetual do design de tipos. Anais do Congresso Internacional de Design da Informação, 9, 917-925.

Disalvo, C. (2012). Adversarial design. Cambridge: The MIT Press.

Dunne, A., Raby, F. (2013). Speculative everything: design, fiction and social dreaming. Cambridge: The MIT Press.

Eira, H., Brandalise, I. (2019). Patadesign: notas pendentes de soluções imaginárias. Brasília: Estereográfica.

Farias, P. L. (2000). Tipografia digital: o impacto das novas tecnologias. 2. ed. Rio de Janeiro: $2 A B$. 
Farias, P.L. (2001). Fontes que não servem para escrever: algumas considerações sobre o status tipográfico dos Dingbat. Revista da ADG, (23), 49-51.

Monteiro Borges, P. (2011, dezembro). Fontes tipográficas digitais: entre a lógica verbal e a gráfico-visual. Galáxia, (22), 262-273.

Rede CoVida - Ciência, Informação e Solidariedade. (2020, maio). Saúde do trabalhador na pandemia de Covid-19: riscos e vulnerabilidades. Disponível em:

$<$ https://redecovida.org/relatorios/saude-do-trabalhador-na-pandemia-de-covid-19-riscos-evulnerabilidades/>. 02/05/2021.

Rocha, R., Atun, R., Massuda, A., Rache, B., Spinola, P., Nunes, L. et al. (2021, April 12). Effect of socioeconomic inequalities and vulnerabilities on health-system preparedness and response to COVID-19 in Brazil: a comprehensive analysis. The Lancet. Disponivel em: $<$ https://www.thelancet.com/journals/langlo/article/PIIS2214-109X(21)00081-4/fulltext>. $13 / 04 / 2021$.

\section{Sobre o(a/s) autor(a/es)}

Antônio Vinícius Dantas Moura de Oliveira, UFRN, Brasil <antonio.v.d.m.o@gmail.com> Eriadne Teixeira do Nascimento, UFRN, Brasil <eritn89@gmail.com> Ewerton Bezerra Siqueira de Miranda, UFRN, Brasil <ewerton.miranda@outlook.com> Rafael de Lima Cardozo, UFRN, Brasil <rafael.cardozo98@gmail.com> Luiza Falcão Soares da Cunha, Dra., UFRN, Brasil <luizafsc@gmail.com> Helena Rugai Bastos, Dra., UFRN, Brasil <helenarugai@gmail.com> 